\title{
Digital 2-Dimensional Photogrammetry Simplified by Using a Marker of a Known Size
}

Jeong Woo Lee ${ }^{1}$, Dong Kyu Kim ${ }^{1}$, Seung Gyun $\mathrm{Chu}^{1}$, Byung Chae Cho ${ }^{1}$, Kyung Young Lee ${ }^{2}$

${ }^{1}$ Department of Plastic and Reconstructive Surgery, Kyungpook National University School of Medicine, Daegu; ${ }^{2}$ Dr. Lee's Aesthetic Surgical Clinic, Daegu, Korea
No potential conflict of interest relevant to this article was reported.
Background A quantitative, rather than visual, assessment of the outcomes of facial surgery has recently become increasingly important, and this is best accomplished using anthropometry. However, most anthropometric methods have limited applicability in office settings.

Methods We conducted preliminary studies on images of a 30-cm straight edge ruler with various camera-to-object distances (OD) and aperture sizes, and identified a convenient range of ODs and the most reliable aperture size of our camera for photogrammetry. Subsequently, we measured various lengths circumjacent to the center of the ruler in the images of different ODs with a graphics viewer program and calibrated the raw data using the central 20-mm length. We obtained a reliable circle in our camera's viewfinder from a chart of percentage differences between the calibrated data and coupled real ruler lengths. Following this, we replaced the concept of the central 20$\mathrm{mm}$ length with a geometric sphere $20-\mathrm{mm}$ in diameter, and applied it to clinical photogrammetry.

Results The percentage differences were almost directly proportional to the real lengths. This value was below $0.48 \%$ in the central $160-\mathrm{mm}$ of all images within an OD range of 50 to $125 \mathrm{~cm}$. In clinical applications, the greatest difference compared to direct measurements was $0.97 \mathrm{~mm}$.

Conclusions Using a graphics viewer program, we performed anthropometric measurements of images that included a sphere of known size, without printing the images; the difference between these and direct measurements was $<1 \mathrm{~mm}$. This method is simple enough for use in an office setting.

Keywords Anthropometry, Photogrammetry, Reference standards

\section{INTRODUCTION}

In this paper, we introduce a digital 2-dimensional (2D) photogrammetry method that utilizes a graphics viewer program to obtain a real metric linear length value from an image in which a marker of a known size is embedded.

Received: Mar 4, 2017 Revised: Jul 25, 2017 Accepted: Aug 3, 2017 Correspondence: Kyung Young Lee Dr. Lee’s Aesthetic Plastic Surgical Clinic, 3F, 2403 Dalgubeol-daero, Suseong-gu, Daegu 42019, Korea.

E-mail: walker3433@naver.com

Copyright @ 2017 The Korean Society for Aesthetic Plastic Surgery.

This is an Open Access article distributed under the terms of the Creative Commons Attribution Non-Commercial License (http://creativecommons.org/licenses/by-nc/4.0/) which permits unrestricted non-commercial use, distribution, and reproduction in any medium, provided the original work is properly cited. $\quad$ www.e-aaps.org
Farkas et al. [1] compared facial measurements obtained from life-size printed photographs with direct measurements and reported reliable results for 20 of the 62 measurements. Nechala et al. [2] placed a ruler beside a subject in a photograph to obtain a reproduction ratio for digital 2D photogrammetry. Those methods are difficult to use to assess the results of various types of craniofacial surgery. Moreover, although 3-dimensional (3D) anthropometric equipment is currently available, it has limited applicability in office settings [3-6]. Many physicians still use direct anthropometry or life-size printed photos [7-9]. Mulliken and Sullivan [10] discussed the importance of including some type of calibration in the images used for photogrammetry, and Price et al. [11] used the subjects' corneal diameter for calibration. However, those authors did not include an assessment of the spherical aberration of the lens in their studies, although this is an important factor in photo- 
grammetry.

This study aimed to obtain a real metric linear length value between 2 points in a non-printed digital image, in which the difference compared to the actual length of the target was $<1 \mathrm{~mm}$ (adopted from the study of Farkas et al. [1]), thereby simplifying the assessment of surgical results in an ordinary office setting. Graphics viewer programs have tools for measuring linear length or angles in a digital photograph. However, the measured linear length is relative, and it is influenced by image resolution, spherical aberration of the lens, and parallax. We hypothesized that a geometric sphere of known size embedded in the digital image could serve as a calibrator for obtaining this length. It is important to remember that unlike direct measurements, a $2 \mathrm{D}$ photograph is an innately distorted relative representation of a real 3D object; therefore, the difference between a photogrammetrically measured value and the real length of a target is due to this relative distortion. For this reason, we used images of a straight-edge ruler, which could be considered as the "absolute" real value. In other words, our experimentation was a process of measuring a ruler. Through these experiments, we attempted to determine the range of our camera's viewfinder, with the difference between the maximum real ruler lengths and the coupled photogrammetrically measured lengths being $<1 \mathrm{~mm}$.

\section{METHODS}

A digital single-lens reflex camera (D80; charge-coupled device [CCD] sensor size, $23.6 \times 15.8 \mathrm{~mm}$; optical viewfinder, $\times 0.94 \mathrm{mag}$ nification; Nikon Corp., Tokyo, Japan) was set up on a tripod with a zoom lens (AF-S DX Zoom-NIKKOR 18-135 mm f/3.5-5.6G IFED; Nikon Corp.). We set our camera to aperture priority mode (f/14), ISO 100, a resolution of 1,936×1,296 pixels (S-resolution), and used the built-in flash. A $30-\mathrm{cm}$ straight edge ruler with a yellow sticker at its center was attached horizontally to a vertical flat wall. The composition of the pictures was set for a landscape shot (i.e., the entire length of the ruler filled the width of the viewfinder, with the focus at the center of the yellow sticker) by adjusting the zoom. All images were obtained with the camera positioned horizontally and the CCD arranged in parallel with the ruler, even for the 2 vertical images. The experimental sequence was as follows: 1 ) environmental factors were assessed; 2) a convenient but reliable camera-to-object distance (OD) range was determined; 3 ) the most reliable aperture size of the camera was determined; 4) the optimum marker size was determined; and 5) the effects of changes in the composition of the photogram were evaluated.

To assess environmental factors, including the pattern of the spherical aberration of our lens, we obtained 2 horizontal and vertical images of the ruler with an OD of $100 \mathrm{~cm}$, and 2 additional images of the reverse arrangement (i.e., the ruler was turned $180^{\circ}$ in the clockwise direction in each position). These 4 images were divided into 15 sequential segments of 20-mm ruler gradations (not 20-mm in length), and each segment was measured using a graphics viewer program (IrfanView version 4.36; Irfan Skiljan, Wiener Neustadt, Austria) [12]. The measured values were subsequently processed as described below (Fig. 1A and 2).

To determine a convenient but reliable OD range, 3 images were obtained with ODs of 50,100, and $150 \mathrm{~cm}$, with an effort to maintain the abovementioned horizontal composition to the extent possible. Each photogram was vertically divided into 7 segments of 20mm gradations (Fig. 1B), and each segment was measured using IrfanView. The measured raw data were calibrated based on the

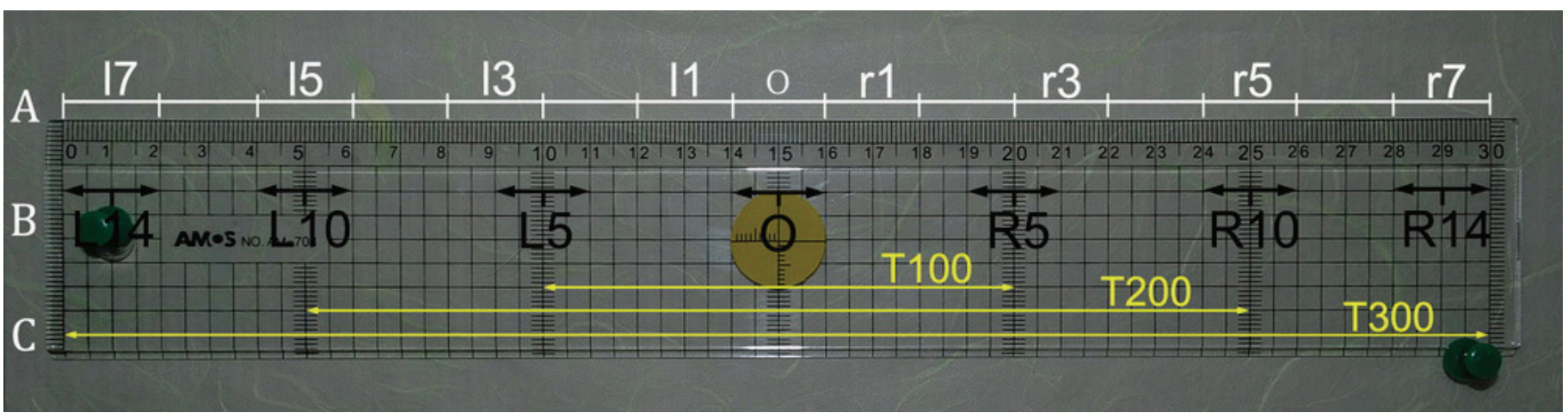

Fig. 1. The full-shot image of a 30-cm long ruler. The upper and lower portions of the original image were trimmed to conserve space. (A) The segments measured in Fig. 2. Central $20-\mathrm{mm}$ gradations on the ruler image (0), the next 20-mm gradations to the left (l1), the next 20-mm gradations to the right ( $\mathrm{r} 1$ ), and so on. (B) The segments measured in Table 1. The central 20 -mm gradations (0), 20-mm gradations, approximately $5 \mathrm{~cm}$ apart, to the left side from the center (L5), 20-mm gradations approximately $5 \mathrm{~cm}$ apart to the right side from the center (R5), and so on. Because the viewfinder of D80 had an optical magnification of $\times 0.94$, both ends of the ruler image filled the full horizontal length of the viewfinder when the photos were obtained. Therefore, the area between L5 and R5 corresponds to the central one-third of the area of the viewfinder. The central yellow sticker was used to facilitate focusing (C) The yellow arrows represent the ranges measured in Fig. 7 to 9 . The 100 -mmruler gradations were measured (T100), and so on. 


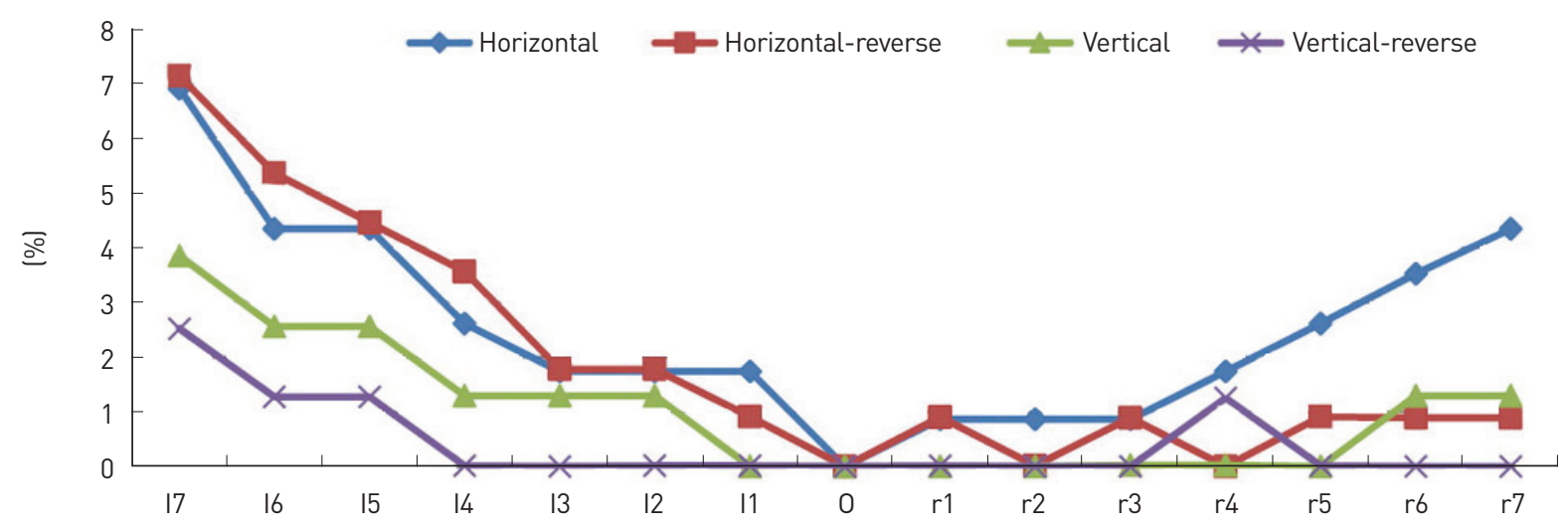

Fig. 2. Chart showing percentage differences. Obtained from the image of a horizontally and vertically attached ruler. Central $20-m m$ gradations of the ruler image (o), the next $20-\mathrm{mm}$ gradations to the left (l1), the next 20-mm gradations to the right ( 1 1), and so on (Fig. 1A). Reverse, an image of the ruler turned $180^{\circ}$ in the clockwise direction.

Table 1. The absolute value of the calibrated difference between the measured value and the real value of each segment was converted into a percentage

\begin{tabular}{|c|c|c|c|c|c|c|c|}
\hline & L14 & L10 & $\mathrm{L}^{\mathrm{el}}$ & $0^{f)}$ & $\mathrm{R}^{\mathrm{gl}^{\mathrm{l}}}$ & R10 & R14 \\
\hline \multicolumn{8}{|l|}{$\mathrm{S} 050^{\mathrm{al}}$} \\
\hline$M(m m)^{b)}$ & 10.25 & 10.08 & 9.99 & 9.91 & 9.91 & 9.99 & 10.24 \\
\hline$C(m m)^{c l}$ & 20.69 & 20.35 & 20.17 & 20.00 & 20.00 & 20.17 & 20.67 \\
\hline$D(\%)^{d)}$ & 3.47 & 1.76 & 0.86 & 0.00 & 0.00 & 0.86 & 3.37 \\
\hline \multicolumn{8}{|l|}{ S100 } \\
\hline$M(m m)^{b)}$ & 10.33 & 10.08 & 9.91 & 9.82 & 9.82 & 9.99 & 10.16 \\
\hline $\mathrm{C}(\mathrm{mm})^{\mathrm{cl}}$ & 21.04 & 20.53 & 20.17 & 20.00 & 20.00 & 20.35 & 20.69 \\
\hline$D(\%)^{d)}$ & 5.18 & 2.64 & 0.87 & 0.00 & 0.01 & 1.73 & 3.45 \\
\hline \multicolumn{8}{|l|}{ S150 } \\
\hline$M(m m)^{b)}$ & 10.25 & 9.99 & 9.74 & 9.57 & 9.65 & 9.74 & 9.91 \\
\hline $\mathrm{C}(\mathrm{mm})^{\mathrm{cl}}$ & 21.43 & 20.89 & 20.35 & 20.00 & 20.18 & 20.35 & 20.71 \\
\hline$D(\%)^{d]}$ & 7.13 & 4.43 & 1.77 & 0.00 & 0.88 & 1.77 & 3.53 \\
\hline
\end{tabular}

a) Resolution 1,936 × 1,296 pixels (S-resolution), camera-to-object distance $=$ $50 \mathrm{~cm}$, and so on.

${ }^{b}$ The measured values of $20-\mathrm{mm}$ ruler gradations at the 7 segments of the images of the ruler with IrfanView Iversion 4.36; Irfan Skiljan, Wiener Neustadt, Austria).

${ }^{c}$ Calibrated values using the known real value of the central $20-\mathrm{mm}$.

${ }^{d}$ The absolute value of the calibrated difference between the measured value and the real value of each segment was converted into a percentage.

${ }^{e}$ The central $20-\mathrm{mm}$ gradations of the ruler image.

fIT The 20-mm gradations approximately $5 \mathrm{~cm}$ apart to the left from the center. ${ }^{9}$ The $20-\mathrm{mm}$ gradations approximately $5 \mathrm{~cm}$ apart to the right from the center, and so on.

known real length value of the central 20-mm, using the following equation (Table 1):

$$
\mathrm{D}=\frac{A}{B} \times C
$$

A, the known real value of the central segment O (20-mm); B,

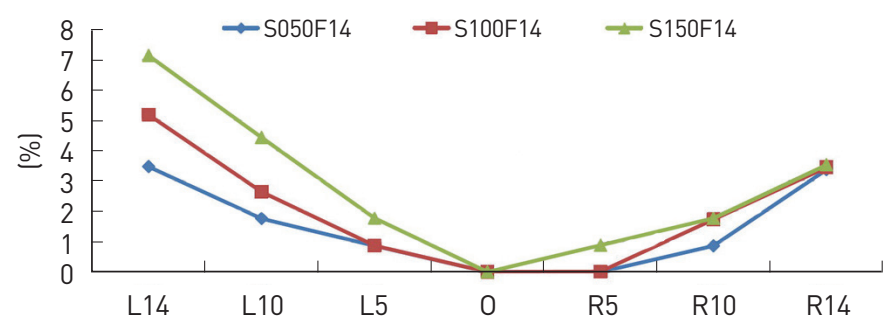

Fig. 3. Chart showing percentage differences. Obtained from images taken with S-resolution (1,936 × 1,296 pixels) and different camerato-object distances (OD). S050F14, S-resolution, OD = 50, aperture size f/14, and so on. The central 20-mm gradations (0), 20-mm gradations, approximately $5 \mathrm{~cm}$ apart to the left side from the center (L5), 20-mm gradations, approximately $5 \mathrm{~cm}$ apart to the right side from the center (R5), and so on.

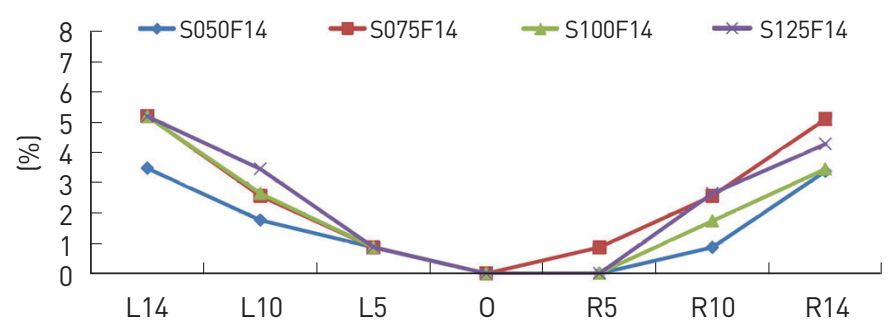

Fig. 4. Chart showing percentage differences. Obtained from images taken with a camera-to-object distance (OD) of 50, 75, 100, and 125 $\mathrm{cm}$. S050F14, resolution 1,936 $\times 1,296$ pixels (S-resolution), OD $=50$ $\mathrm{cm}$, aperture size $\mathrm{f} / 14$, and so on. The central 20-mm gradations (0), 20-mm gradations, approximately $5 \mathrm{~cm}$ apart to the left side from the center (L5), $20-\mathrm{mm}$ gradations, approximately $5 \mathrm{~cm}$ apart to the right side from the center (R5), and so on.

the measured value of segment $\mathrm{O}(\mathrm{mm})$; $\mathrm{C}$, the measured value of each segment ( $\mathrm{mm})$; D, the calibrated value of each segment $(\mathrm{mm})$.

We used Excel 2010 (Microsoft Corp., Redmond, WA, USA) for the calculations and for generating the chart figures. The absolute 
differences between the calibrated values and real values of the coupled segments were converted into percentages; these data are presented in charts (Fig. 3 and Table 1).

We processed 2 images with ODs of 50 and $100 \mathrm{~cm}$ and 2 additional images with ODs of 75 and $125 \mathrm{~cm}$, as described above (Fig. 4).

To determine the most reliable aperture size of the lens, we obtained 2 additional photographs $(\mathrm{OD}=100 \mathrm{~cm}$; aperture size, $\mathrm{f} / 5.6$ and $f / 36$, which are at the extreme ends of the $f$-numbers). The 3 photographs with the $f / 5.6, f / 14$, and $f / 36$ aperture sizes were processed as described above (Fig. 5). Based on this chart, we found that the data obtained from the photographs with an aperture size of $\mathrm{f} / 14$ were the most reliable. Therefore, we selected 6 additional circumjacent f-numbers (f/10, f/11, f/13, f/16, f/18, and f/20) and took 6 additional photographs, with an $\mathrm{OD}$ of $100 \mathrm{~cm}$. We processed the 7 photographs as described above (Fig. 6). The 7 curves in Fig. 6 show that the curves of the $f / 13, f / 14$, and $f / 16$ sizes had relatively low percentage differences and better symmetry. Thus, we took 8 additional photographs $(\mathrm{OD}=50,75,100$, and $125 \mathrm{~cm}$; aperture

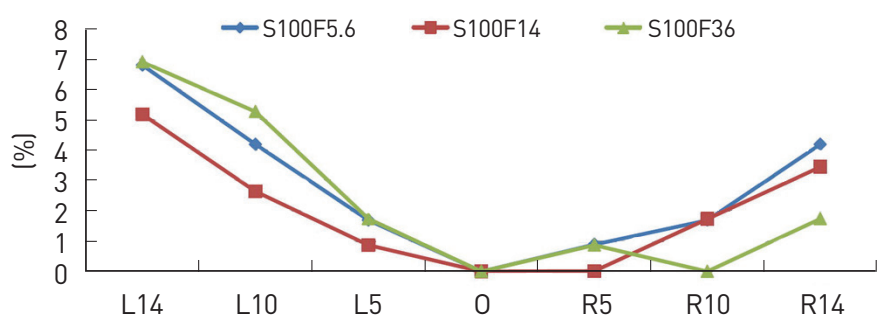

Fig. 5. Chart showing percentage differences. Obtained from images taken with aperture sizes of $\mathrm{f} / 14$ and both extreme f-numbers. S100F5.6, resolution 1,936 × 1,296 pixels (S-resolution), camera-to-object distance $=100 \mathrm{~cm}$, aperture size $\mathrm{f} / 5.6$, and so on. The central $20-\mathrm{mm}$ gradations (0), 20-mm gradations, approximately $5 \mathrm{~cm}$ apart to the left side from the center (L5), 20-mm gradations, approximately 5 $\mathrm{cm}$ apart to the right side from the center (R5), and so on.

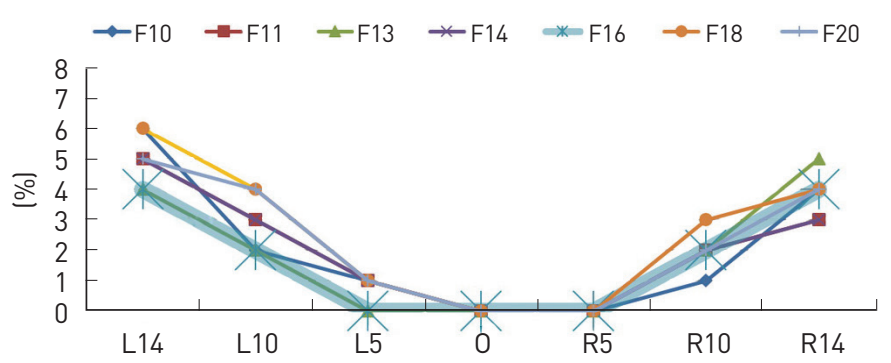

Fig. 6. Chart showing percentage differences. Obtained from images taken with a camera-to-object distance $=100 \mathrm{~cm}$, aperture size of $\mathrm{f} / 14$, and 6 circumjacent $f$-numbers. Among the 7 curves, that of $f / 16$ was the most beneficial with respect to the magnitude and symmetry of the differences. The central $20-\mathrm{mm}$ gradations (0), 20-mm gradations, approximately $5 \mathrm{~cm}$ apart to the left side from the center (L5), 20-mm gradations, approximately $5 \mathrm{~cm}$ apart to the right side from the center (R5), and so on. size, $f / 13$ and $f / 16$ ). We grouped the 12 photographs by the f-number and measured 15 circumjacent ranges of the center $(20,40,60$, $80,100,120$, etc., through 300 -mm gradations) from the 4 images with an OD of 50, 75, 100, and $125 \mathrm{~cm}$ in each group (Fig. 1C). We processed the obtained data as described above, and we generated charts of the percentage differences, and found that the chart for the aperture size $f / 16$ showed the lowest and most regular pattern of difference (Fig. 7).

To determine the most favorable size of the marker with the $f / 16$ image $(\mathrm{OD}=100 \mathrm{~cm})$, we measured each of the $30-\mathrm{mm}$ ruler gradations circumjacent to the 7 dividing points with IrfanView, calibrated them using the known real value of the central 10, 20, and $40 \mathrm{~mm}$ as hypothetical calibrators, and generated a chart of the percentage differences (Fig. 1B and 8). We changed the compositions of the photograph as follows: one was changed to have the entire length of the ruler occupying the central two-thirds of the width of

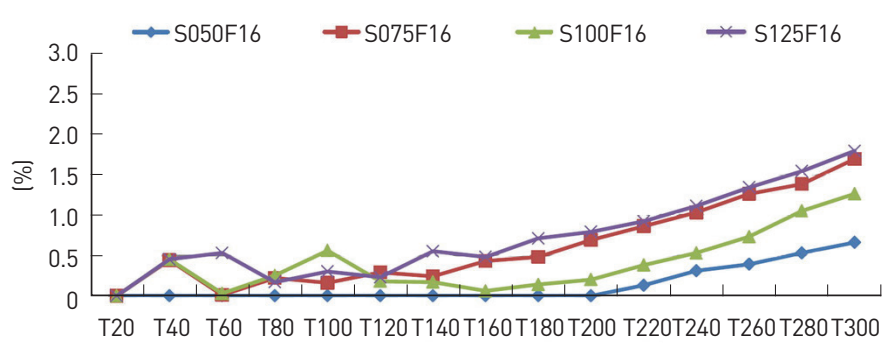

Fig. 7. Chart showing percentage differences. Obtained by measuring various ranges circumjacent to the center (Fig. 1C, calibrated with the central $20-\mathrm{mm}$ segment of the ruler) from images taken with an aperture size of $f / 16$. S050F16, resolution, 1,936 $\times 1,296$ pixels (S-resolution); camera-to-object distance $=50 \mathrm{~cm}$; aperture size $\mathrm{f} / 16$; the percentage difference ([calibrated measured value minus real value of the measured target]/[real value of the measured target] $\times 100[\%]]$ of the central $20-\mathrm{mm}$ gradations (T20); the central 40-mm gradations (T40); and so on. The highest percentage difference of T160 was $0.48 \%(160 \mathrm{~mm} \times 0.48 \%=0.768 \mathrm{~mm})$ and that of $\mathrm{T} 180$ was $0.71 \%(180 \mathrm{~mm} \times 0.71 \%=1.278 \mathrm{~mm})$. T160 also corresponds to a circle in the central 160/300 of the viewfinder. Within an image, it corresponds to a circle in the central $50 \%([160 / 300] \times 0.94$ optical magnification of the viewfinder) of the image.

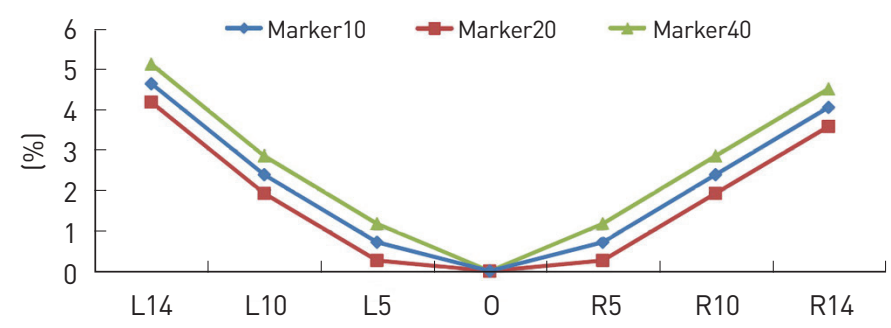

Fig. 8. Chart showing percentage differences. Obtained from images taken with different hypothetical marker sizes. Central 10-mm ruler gradations as a hypothetical marker (Marker10), and so on. 
the viewfinder, and the other was changed to have the central onethird shown with a higher resolution of 3,872 $\times 2,592$ pixels (L-resolution) to obtain a clear enlarged image of the ruler to measure. From those 2 images, we also measured 15 circumjacent ranges of the center and generated a chart of the percentage differences (Fig. 1C and 9).

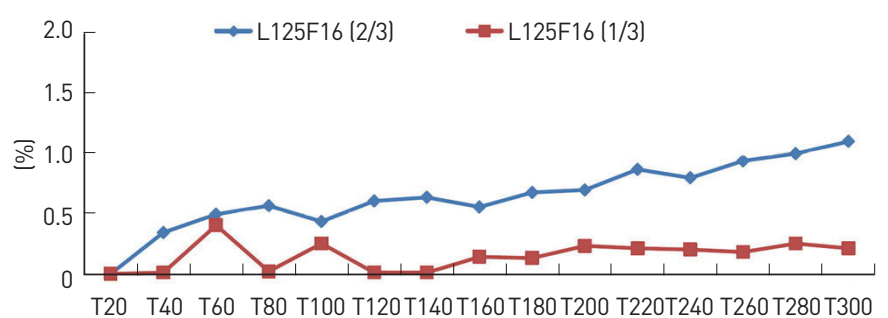

Fig. 9. Chart showing percentage differences. Obtained from the 2 changed compositions of the ruler image. L125F16 (2/3), resolution $3,872 \times 2,592$ pixels (L-resolution), camera-to-object distance $=125$ $\mathrm{cm}$, aperture size $\mathrm{f} / 16$, the composition of the entire image of the ruler occupying the central 2/3 width of the viewfinder. L125F16 (1/3), the composition of the ruler occupying the central $1 / 3$ of the viewfinder.
Finally, we generated a plastic geometrical sphere $20-\mathrm{mm}$ in diameter and attached it to a $15-\mathrm{cm}$ long bamboo stick (like a lollipop); thereafter, the concept of the central 20-mm of the ruler was replaced with the sphere, and the ruler was replaced with a face. While holding the camera in both hands, we took 5 photographs of a subject on whom the spherical marker was placed at unknown ODs within the 50 to $125 \mathrm{~cm}$ range (over-the-desk width), at f/16, ISO 100 (ISO auto maximum sensitivity 400), with the focus on the sphere. We composed the measuring area within the central $1 / 2$ of the viewfinder with the aid of the viewfinder grids. Subsequently, we obtained the absolute differences between the measurements that were performed using the method described above from these 5 sphere-embedded images and direct measurements of the periorbital area of the same subject (Fig. 10 and Table 2). Before measuring the landmarks, the interpupillary line was arrayed horizontally, and adequate digital magnification of the image was attained using IrfanView; thereafter, to measure the diameter of the sphere, the boundary of the sphere was selected as an exact rectangle, and the length of a side of a square was measured (Fig. 10). Direct measurement was performed using the projective method with Vernier calipers, up to units of $0.5 \mathrm{~mm}$, the smallest unit of the caliper.

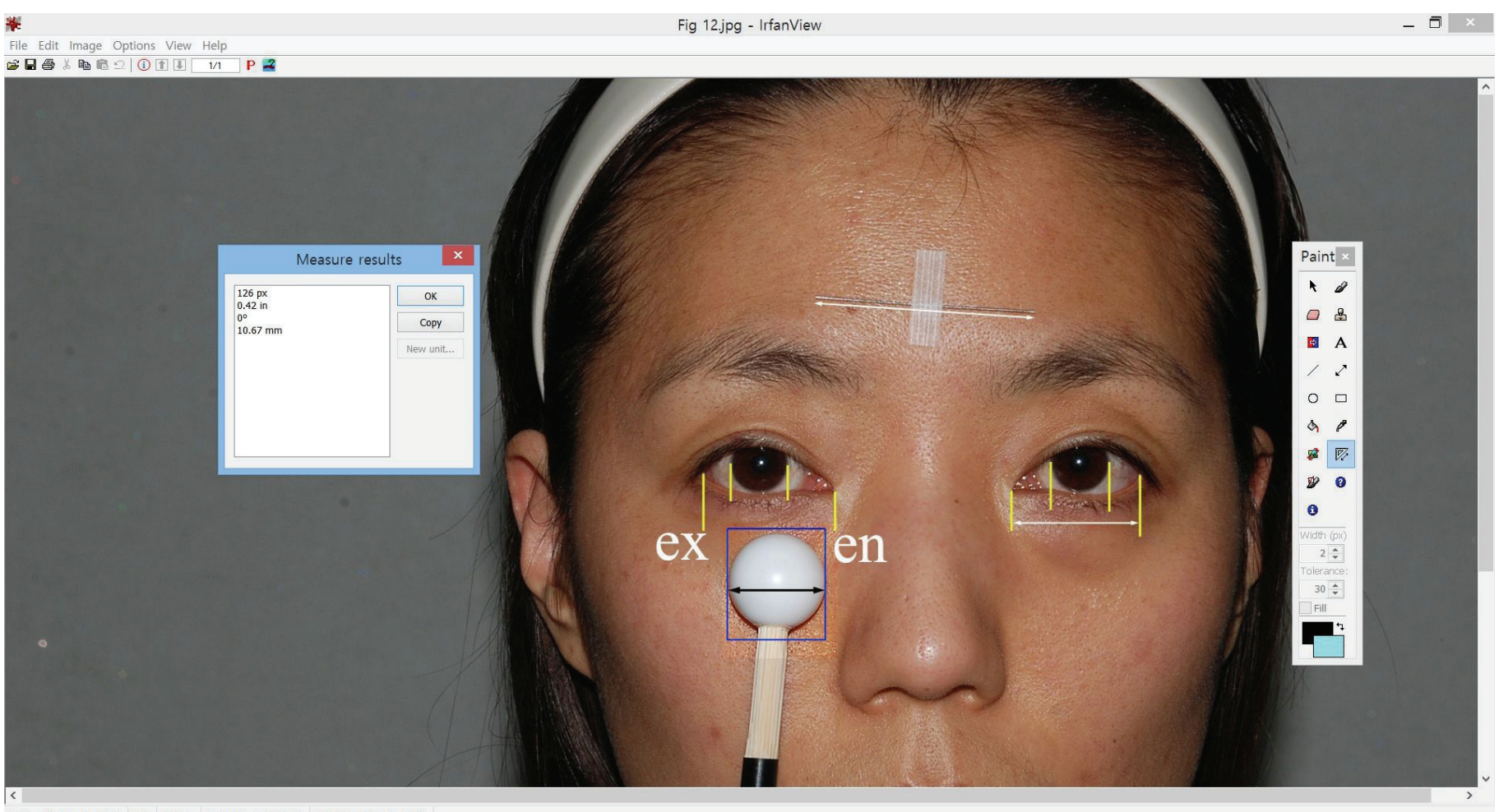

$\begin{array}{lllll}1936 \times 1296 \times 24 \text { BPP } & 1 / 1 & 100 \% & 1.33 \text { MB / 7.18 MB } \quad 2014-01-08 / 22: 49: 06\end{array}$

Fig. 10. Screenshot of clinical digital photogrammetry using IrfanView (version 4.36; Irfan Skiljan, Wiener Neustadt, Austria). The metal rod on the forehead is an artificial landmark for checking our method of photogrammetry and our skill level of direct measurement in the early stages of our experiment. The black arrow represents the diameter of the sphere, which is displayed in the window of 'Measure Results'. ex, exocanthion; en, endocanthion. 
Table 2. Differences between direct and photogrammetric measurements obtained from 5 clinical images of the same subject with S-resolution $(1,936 \times 1,296$ pixels) and random camera-to-OD from 50 to $125 \mathrm{~cm}$

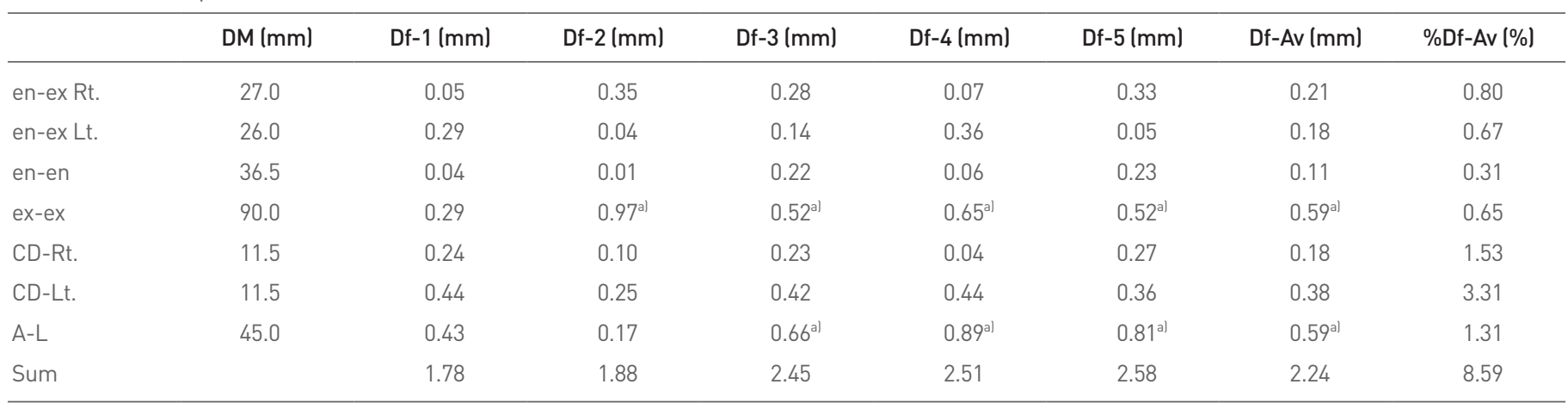

OD, object distances; en, endocanthion; ex, exocanthion; Rt., right; Lt., left; CD, corneal diameter; A-L, artificial landmark of the steel rod; DM, value of the direct measurement; Df, the difference; Df-Av, the average of $5 \mathrm{Df} ; \% \mathrm{Df}-\mathrm{Av}$, the percentage difference of Df-Av.

al The difference values are $>0.5 \mathrm{~mm}$. The image of Df- 1 had a longer OD and that of Df- 5 had a shorter OD.

\section{RESULTS}

The percentage differences were higher on the left and upper sides of the images and were remarkably lower in the vertical images (Fig. 2).

All calibrated values fell into a very narrow range, with the leftside and peripheral values being larger (Table 1). However, the magnitude of the differences stood out after the data were converted to percentages. The charts of the percentage differences in the ODs showed a similar symmetric pattern of curves dispersing peripherally within the 50 to $125 \mathrm{~cm}$ OD range (Fig. 4). The most regular and lowest differences were found for the $\mathrm{f} / 16$ images (Fig. 5 and 6). The highest percentage difference was $0.48 \%(160 \times 0.48 \%=$ $0.77 \mathrm{~mm})$ for the $160-\mathrm{mm}$ length and $0.71 \%(180 \times 0.71 \%=1.28$ $\mathrm{mm}$ ) for the $180-\mathrm{mm}$ length, and the percentage differences and measured target lengths around the center were almost directly proportional to each other (Fig. 7).

Among the 3 hypothetical marker sizes, the $20-\mathrm{mm}$ size showed the least differences (Fig. 8).

In the images with altered compositions, the magnitudes of the percentage differences showed a remarkable distinction between the 2 compositions (Fig. 9).

In clinical applications, differences greater than $0.5 \mathrm{~mm}$ were observed for measurements of the inter-exocanthion and artificial landmark lengths, and the highest value was $0.97 \mathrm{~mm}$ (Table 2).

\section{DISCUSSION}

Our hypothesis that a geometric sphere of known size embedded in a digital image can serve as a calibrator for obtaining the length between 2 points in the image was proven in this study. In the clinical application, the greatest difference between photogrammetric measurements and direct measurements was $0.97 \mathrm{~mm}$ (Fig. 10 and Table 2).

Theoretically, within the central half-circular area $(160 / 300 \times$
$0.94=0.50$ ) of an image taken by our camera lens, set with an aperture size of $f / 16$, focused on the marker, and an OD between 50 and $125 \mathrm{~cm}$, a target length of up to $160 \mathrm{~mm}$ could be measured, with a difference of $<1 \mathrm{~mm}$ (Fig. 7). In other words, the central half (160/300, more exactly) area of the width of the viewfinder was the theoretical reliable circle of our camera with the lens. Considering that the maximum breadth of a subject, between the eurion to eurion in adult males, is on average $153 \mathrm{~mm}$ [1], this length limit would be an adequate range for assessing the results of facial surgery. In the study of Farkas et al. [1], the most important distortion and limitation were found in the measurements between points at the distant coronal and sagittal levels in the frontal and profile view, respectively. This finding could be explained by the spherical aberration and reducible by changing the composition of a photograph with a higher resolution, as shown in Fig. 9.

In clinical applications, to decrease this difference, the calibrator should be moved as close as possible to the target to be measured. Moreover, as in other types of photogrammetry, the importance of using a photographic technique that does not generate parallax to the target structure must be emphasized. For the corneal diameter, the percentage difference was higher on the left side than on the right side, because the calibrator was positioned on the right side of the image (Table 2).

In 1949, Tanner and Weiner [13] suggested the importance of photogrammetry in medical assessments, which was followed by similar reports $[1,2,14,15]$. The importance of some type of calibration in the image was emphasized by Mulliken and Sullivan [10]. The results of facial surgery can be assessed anthropometrically and clearly summarized in tabular form in order to present a large number of operated cases. We think that this is the principal point of importance that was suggested by many authors who studied various kinds of anthropometric measurements. The diameter of a geometric sphere, unlike a flat ruler [2], does not fluctuate according to the viewing angle; therefore, it is an adequate calibrator. Price 


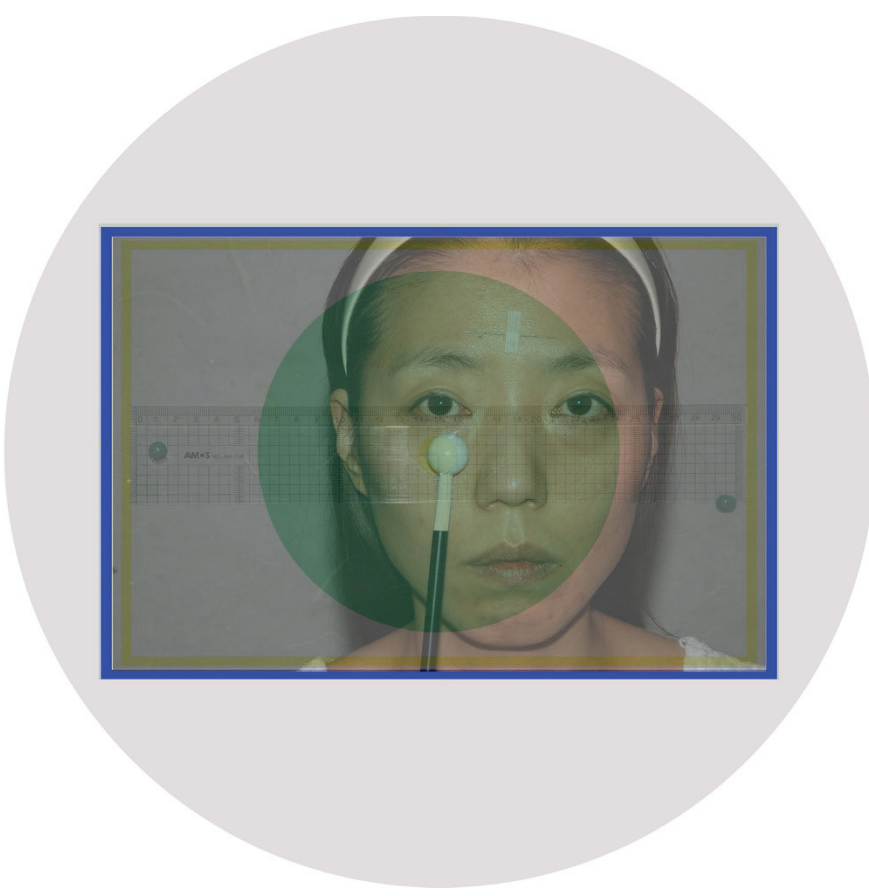

Fig. 11. Schematic presentation of our study. Outer circle, the image circle produced by the lens; outer blue rectangle, the charge-coupled device (CCD) sensor of the camera body; inner yellow rectangle, outline of the viewfinder; the ruler image; and the central green circle, the reliable circle of our camera and lens measurable up to a $160-\mathrm{mm}$ length of the target with a difference $<1 \mathrm{~mm}$; Fig. 10 is superimposed on the CCD sensor.

et al. [11] used the subjects' corneal diameter for calibration, but that technique has limited applicability in terms of position and viewing angle, and has individual differences. Our sphere, which was 20-mm in diameter (range of fabrication error, $1 / 100 \mathrm{~mm}$ ), was generated with the help of a factory that conducts micro-milling operations.

The spherical aberration of the lens we used was slightly higher towards the left and upper sides, but the magnitude of difference between each coupled image of the reversed object position could have been caused by measurement errors, inaccuracies in the photography technique, and even by the ruler itself (Fig. 2). The noticeable difference between the horizontal and vertical images (Fig. 2 ) and the results of the changed composition of Fig. 9 were anticipated when considering the relationship between the image circle of a lens and the CCD sensor of a camera (Fig. 11).

In the study of Farkas et al., the OD chosen to reduce photographic error was 11 feet (i.e., $3.35 \mathrm{~m}$ ) [1], which is too large for the assessment of surgical results. The OD range in our study (50-125 $\mathrm{cm}$ ) is convenient for obtaining clinical photographs in an office setting, with no limitation in the angle of view with the sphere.

Among the 3 tested hypothetical marker sizes, the most favorable result was obtained for the 20-mm size (Fig. 8). However, the theoretical basis for this finding could not be explained. Additively, with sequential images, the excursion of a landmark would also be measurable.

Our method could be used as a technique for determining the reliable circle of a photography system for photogrammetry. The reliable circle would be expected to be wider in a higher quality lens with lower magnitude and further symmetricity of its spherical aberration, and especially with respect to the width of the image circle (Fig. 11). IrfanView is an inexpensive but fast and compact program that has many useful tools for photogrammetry [12].

Further, more refined investigations with various camera bodies and lenses are required. A summary of the findings of this study is provided in Fig. 11.

\section{ACKNOWLEDGMENT}

This article was presented as a poster at the 2014 international meeting of the Korean Society of Plastic and Reconstructive Surgeons on November 7-9, 2014 in Seoul, Korea. We are grateful to Irfan Skiljan in Austria, who developed the graphics viewer program, IrfanView, and provided it as freeware for private and non-commercial use and at a non-commercial cost for commercial use. Further, we are grateful to Kyung Il Lee, the President of Kumkang Precision Co. in Daegu, Korea, who facilitated our development of the spherical marker.

\section{PATIENT CONSENT}

Patients provided written consent for the use of their images.

\section{REFERENCES}

1. Farkas LG, Bryson W, Klotz J. Is photogrammetry of the face reliable? Plast Reconstr Surg 1980;66:346-55.

2. Nechala P, Mahoney J, Farkas LG. Digital two-dimensional photogrammetry: a comparison of three techniques of obtaining digital photographs. Plast Reconstr Surg 1999;103:1819-25.

3. Ferrario VF, Sforza C, Colombo A, et al. Morphometry of the orbital region: a soft-tissue study from adolescence to mid-adulthood. Plast Reconstr Surg 2001;108:285-92; discussion 93.

4. Landes CA, Trolle M, Sader R. Rapid 3-dimensional anthropometry by semiautomatic data acquisition and processing. Ann Plast Surg 2007; 58:698-703.

5. Heike CL, Cunningham ML, Hing AV, et al. Picture perfect? Reliability of craniofacial anthropometry using three-dimensional digital stereophotogrammetry. Plast Reconstr Surg 2009;124:1261-72.

6. Tanna N, Wan DC, Kawamoto HK, et al. Craniofacial microsomia soft-tissue reconstruction comparison: inframammary extended circumflex scapular flap versus serial fat grafting. Plast Reconstr Surg 2011;127:802-11. 
7. Park DH, Choi WS, Yoon SH, et al. Anthropometry of asian eyelids by age. Plast Reconstr Surg 2008;121:1405-13.

8. Lee CT, Garfinkle JS, Warren SM, et al. Nasoalveolar molding improves appearance of children with bilateral cleft lip-cleft palate. Plast Reconstr Surg 2008;122:1131-7.

9. Farahvash MR, Khak J, Horestani MJ, et al. Facial aesthetic analysis in beautiful Persian female subjects aged 13 to 30 years by means of photogrammetry. Plast Reconstr Surg 2010;125:245e-7e.

10. Mulliken JB, Sullivan SR. Discussion. Nasoalveolar molding improves long-term nasal symmetry in complete unilateral cleft lip-cleft palate patients. Plast Reconstr Surg 2009;123:1007-9.

11. Price KM, Gupta PK, Woodward JA, et al. Eyebrow and eyelid dimen- sions: an anthropometric analysis of African Americans and Caucasians. Plast Reconstr Surg 2009;124:615-23.

12. Irfan Skiljan. IrfanView version 4.36. Wiener Neustadt, AT: Irfan Skiljan, 2011. [cited by 2017 Apr 5]. Available from: http://www.irfanview.com/.

13. Tanner JM, Weiner JS. The reliability of the photogrammetric method of anthropometry, with a description of a miniature camera technique. Am J Phys Anthropol 1949;7:145-86.

14. Gavan JA, Washburn SL, Lewis PH. Photography: an anthropometric tool. Am J Phys Anthropol 1952;10:331-53.

15. Davidson TM. Photography in facial plastic and reconstructive surgery. J Biol Photogr Assoc 1979;47:59-67. 